\title{
Pulmonary Infection Due to Mycobacterium malmoense in a Patient with Crohn Disease
}

\author{
Cory D Cowan, John J Hawboldt, and Mazen Bader
}

\section{INTRODUCTION}

$\mathrm{T}$ The incidence of nontuberculous mycobacteria (NTM) infections has increased over the past couple of decades, the most common clinical manifestation being pulmonary disease. ${ }^{1}$ Although Mycobacterium avium complex continues to be responsible for most cases, a number of other species may cause infection, ${ }^{1}$ including Mycobacterium malmoense, a slow-growing nonphotochromogenic mycobacterium that was first isolated in $1977 .^{2}$ Although relatively common in the United Kingdom and northern Europe, M. malmoense has to date been isolated only rarely in North America. This species presents unique challenges for diagnosis and treatment and continues to cause considerable morbidity and mortality if inadequately treated. ${ }^{1,3}$ The following case report describes a pulmonary infection caused by $M$. malmoense in a middle-aged Canadian woman* from Newfoundland and Labrador.

\section{CASE REPORT}

In December 2006, a 53-year-old woman was referred to the Eastern Health Infectious Diseases clinic for evaluation of an unresolved cavitary lesion in the right upper lobe. Upon presentation she had dry cough, dyspnea both at rest and with exertion, fatigue, night sweats, poor appetite, and inability to gain weight. She denied any fever or chest pain. Nausea, early satiety, and mild diarrhea were present. She had no visual symptoms. The patient's medical history included longstanding Crohn disease (for more than 20 years), iritis, osteoporosis, and hypothyroidism. Her medications at the time were Pentasa (mesalamine) $2 \mathrm{~g}$ PO qid, alendronate $70 \mathrm{mg}$ PO weekly, Ostoforte (ergocalciferol) once every 2 weeks, TUMS (regular-strength calcium carbonate) 3 tablets once daily, levothyroxine $50 \mu \mathrm{g}$ PO once daily, and esomeprazole $40 \mathrm{mg}$ PO once daily. She was a housewife, and the only notable travel history was a trip to Florida several years previously. The

\footnotetext{
*To protect the privacy of the individual described here and the family, all unique identifying information not pertinent to the case has been omitted from this report.
}

patient had a significant smoking history (2 packs/week for more than 30 years), but she denied any use of ethanol or illicit drugs.

More than a year earlier, in July 2005, the patient had presented to the emergency department at the Health Sciences Center in St John's with a few weeks' history of dry cough, progressive dyspnea, low-grade fever, weight loss, and progressive fatigue. At that presentation, chest radiography indicated infiltrate in the right upper quadrant. Subsequent computed tomography (CT) of the chest revealed a cavitary lesion measuring $4 \times 2.8 \mathrm{~cm}$ with a surrounding nodular infiltrate. Bronchoscopy indicated that the main and lobar bronchi were normal. Bronchoalveolar lavage (BAL) was performed, and the fluid was sent for routine bacterial, fungal, and acid-fast staining with culture. The smear from the BAL was positive for acid-fast bacilli, but the cultured specimen failed to grow. On the basis of the patient's symptoms, the positive acid-fast bacilli smear, and a positive $11-\mathrm{mm}$ induration on tuberculin skin testing, it was decided to initiate treatment for tuberculosis, consisting of isoniazid $900 \mathrm{mg}$ PO 2 times weekly, rifampin $600 \mathrm{mg}$ PO 2 times weekly, pyrazinamide $2.5 \mathrm{~g}$ PO 2 times weekly, and vitamin B6 $25 \mathrm{mg}$ PO daily for 6 months.

After 4 months of this treatment, there was mild improvement in the patient's symptoms. Repeat chest CT (in November 2005) revealed no change in the size of the cavitary lesion in the right upper lobe. The patient then underwent CT-guided percutaneous needle biopsy and aspiration of the cavitary lesion. The specimen was sent for routine bacterial, fungal, and mycobacterial staining, culture, and cytology, but this testing did not suggest a specific diagnosis. The patient finished the 6-month course of anti-tuberculosis therapy, at which point it was decided to follow the case clinically with chest CT every 3 to 6 months without treatment.

In late October 2006, follow-up chest CT revealed that the cavitary lesion in the right upper lobe had increased in size and had spread to the left lower lobe. The diameter of the lesion now measured $4.6 \mathrm{~cm}$, with a $7-\mathrm{mm}$-thick wall. Satellite lesions were apparent throughout the right lung, and several nodular densities were noted within the left lower lobe. Despite saline 
induction, the patient was unable to provide a lower respiratory tract specimen, and she therefore underwent repeat bronchoscopy with BAL. The airways were normal, without evidence of inflammation. Both bacterial and fungal staining and culture were negative. However, auramine-rhodamine staining of the BAL specimen yielded a strong positive reaction (3+) for acid-fast bacilli, and polymerase chain reaction was negative for Mycobacterium tuberculosis. Within a few weeks, the cultured specimen from BAL started to grow mycobacteria. However, testing with nucleic acid probes was negative for $M$. tuberculosis and Mycobacterium avium-intracellulare. The specimen was sent to the National Microbiology Laboratory (Winnipeg, Manitoba) for isolation and identification of the pathogen. Early results indicated an organism similar to Mycobacterium porcinum, but further testing was needed. The patient's condition was relatively stable at this time, and it was decided to start a course of clarithromycin with ciprofloxacin until evaluation by an infectious diseases specialist.

Upon further examination at the Eastern Health Infectious Diseases clinic in December 2006, the patient's vital signs were stable, she was afebrile, and there was no hemoptysis. The patient was cachetic with obvious tachypnea, but there were no signs of respiratory distress. On auscultation, the clinician noted decreased breath sounds and scattered crackles in the right lung. The results of an abdominal examination were normal except for mild epigastric tenderness. Significant laboratory findings included partial pressure of carbon dioxide $15 \mathrm{mmol} / \mathrm{L}$ (normal range $22-32 \mathrm{mmol} / \mathrm{L}$ ), creatinine $110 \mu \mathrm{mol} / \mathrm{L}$ (normal range $35-88 \mu \mathrm{mol} / \mathrm{L}$ ), albumin $23 \mathrm{~g} / \mathrm{L}$ (normal range 33-53 g/L), and hemoglobin $117 \mathrm{~g} / \mathrm{L}$ (normal range $120-160 \mathrm{~g} / \mathrm{L}$ ). Leukocyte counts and liver function were normal. During this visit, it was decided to add imipenem to her regimen for suspected infection with NTM.

In January 2007, the National Microbiology Laboratory returned the culture results, identifying the organism (using hsp65 sequencing) as $M$. malmoense sensitive to rifabutin, clarithromycin, and amikacin. After discussion with a European expert regarding the treatment of $M$. malmoense infections, and in consultation with the patient's ophthalmologist, it was decided to start clarithromycin $500 \mathrm{mg}$ PO bid, rifampin $600 \mathrm{mg}$ PO once daily, and ethambutol $800 \mathrm{mg}$ PO once daily. On the basis of available evidence and given the presence of comorbidities (e.g., Crohn disease, cachexia), the intended duration of treatment was a minimum of 18 to 24 months. Close monitoring of iritis was performed regularly by the ophthalmologist. The patient underwent monthly follow-up at the Eastern Health Infectious Diseases clinic to assess adherence and response to therapy; this follow-up included repeated interventions to increase her weight. During this treatment, the patient's dyspnea, fatigue, and night sweats improved, but she was unable to gain weight despite supplementary nutritional support. Repeat chest CT at the end of February 2007, after a few months of treatment, showed that the size of the cavitary lesion was stable, with thinning of the wall and no new nodular infiltrate. There was no evidence of worsening of the iritis or adverse hematologic and hepatic reactions during the treatment, and she continued with the prescribed medication regimen.

In October 2007 she presented to the emergency department with several days' history of productive cough, worsening dyspnea, fever, and chills. Chest CT suggested a new air-fluid level in the cavity, with surrounding new airspace infiltrate. Superimposed community-acquired pneumonia was diagnosed, and a course of piperacillin-tazobactam and moxifloxacin was started. Unfortunately, the patient's condition progressed to respiratory failure requiring intubation and mechanical ventilation. Bronchoscopy revealed purulent secretions in the right lobar bronchus, mainly in the upper lobe. The patient's condition continued to worsen despite broad-spectrum antimicrobials and supportive care. There was no response to these interventions, and family members and the attending physicians decided to provide comfort care only. Culture of BAL specimens revealed no organisms, and both smear and culture for acid-fast bacilli were negative.

\section{DISCUSSION}

Mycobacterium malmoense was first described in 1977 by Schröder and Juhlin on the basis of 4 cases of pulmonary infection in the city of Malmo, Sweden, from which the organism gets its name. ${ }^{2}$ Most published case reports have originated in northern Europe and the United Kingdom; however, several reports, most from the United States, have identified $M$. malmoense as a causative pathogen in North America. ${ }^{4,5}$ To the authors' knowledge, this is only the second documented case of pulmonary infection with $M$. malmoense in Canada, the other having occurred in western Canada (specifically, British Columbia). ${ }^{6}$ Interestingly, in a review of more than 20000 pulmonary NTM isolates between 1997 and 2003 in Canada's largest province (Ontario), no cases of $M$. malmoense were identified. ${ }^{7}$ Nonpulmonary cases of NTM infection may also occur, and investigators from Nova Scotia recently reported the first 2 Canadian cases of cervical adenitis due to $M$. malmoense in their 10-year review of NTM adenitis in the province. ${ }^{8}$ It remains unclear how the middleaged woman described in the current report came into contact with this particular pathogen; however, it was most likely through an environmental source, as the organism is commonly found in soil and water. ${ }^{1}$ To date, there is no evidence of animal-to-human or human-to-human transmission of NTM disease.

Like other NTM species, $M$. malmoense causes pulmonary infection in most cases. It has also been responsible for lymphadenitis (particularly cervical adenitis in children, as observed in Nova Scotia ${ }^{8}$ ), skin and soft-tissue infections, or, in rare cases, disseminated disease. ${ }^{1,9}$ Pulmonary infection due to NTM most commonly arises in patients with coexisting structural lung disease such as chronic obstructive pulmonary 
disease, bronchiectasis, cystic fibrosis, previous tuberculosis, and occupational exposure (e.g., pneumoconiosis). ${ }^{1}$ Nodular NTM associated with bronchiectasis typically afflicts postmenopausal women with a characteristic morphotype (thin body habitus), including those with scoliosis, pectus excavatum, joint hypermobility, and prolapse of the mitral valve. ${ }^{1}$ Inhibitors of tumour necrosis factor $\alpha$ (e.g., infliximab, adalimumab, etanercept) may pose problems through their association with the development of active tuberculosis in those with latent infection and a predisposition to invasive fungal infections. ${ }^{1}$ The risk of development or progression of active NTM infections posed by these agents is still unknown.

Pulmonary infection due to $M$. malmoense often presents with variable and nonspecific symptoms, including cough, dyspnea, hemoptysis, sputum production, malaise, and weight loss. ${ }^{1}$ Updated recommendations for the diagnosis and treatment of NTM diseases were released in $2007,{ }^{1}$ and although there is limited evidence regarding the specific management of $M$. malmoense disease, many of the general NTM recommendations may still be applicable. Table 1 outlines the clinical and microbiologic information required to assess and diagnose NTM pulmonary disease. For diagnosis, patients must meet both clinical criteria and one or more microbiologic criteria. To ensure that appropriate treatment is given, confirmatory culture to identify the specific pathogen is recommended, since certain standard antituberculosis medications (e.g., isoniazid) are ineffective against $M$. malmoense. ${ }^{1,10,11}$ Laboratory diagnosis of this pathogen still begins with an acid-fast bacilli smear; however, the advent of liquid culture systems has shortened the time required to detect NTM species, and these systems offer improved sensitivity. Molecular tools such as $16 \mathrm{~S}$ rDNA gene sequencing and highperformance liquid chromatography can also be used, but they are costly and technically demanding. Line probe assays that evaluate DNA probes may also be useful. Regardless of the method used, one must still keep in mind that up to 8-12 weeks may be required to grow the organism in the laboratory.

Once NTM disease is diagnosed, prompt treatment is recommended for most patients. The optimal chemotherapy regimen is still unknown; however, most strains of $M$. mal- moense are sensitive to ethambutol, and some are sensitive to rifampicin. As for other NTM species, many authors have reported that the clinical response to treatment does not correlate well with standard in vitro testing for antimicrobial susceptibility. ${ }^{1,12,13}$ Although overall cure rates remain below $50 \%$, the best responses have been observed with combinations of isoniazid, rifampin, and ethambutol, with or without fluoroquinolones and macrolides. ${ }^{5,6,11,13-15}$ In 2 retrospective studies, it was noted that patients treated for 18-24 months with regimens that included ethambutol and rifampicin did better than those treated with other regimens or for shorter durations. ${ }^{13,14}$ In 1985, Banks and others ${ }^{13}$ published one of the first treatment reviews, which covered 37 patients from Wales from whom M. malmoense had been isolated, 34 of whom had significant pulmonary disease and all of whom had cavitating lesions revealed by chest radiography. Pre-existing lung disease was present in 22 of the patients, and another 4 were taking immunosuppressive drugs. Various drugs were used. The 5 patients who received a standard drug regimen consisting of isoniazid, rifampicin, and ethambutol achieved a "complete response", but only after 18-24 months. ${ }^{13}$ Relapse occurred in 3 of another 5 patients who were treated with the same drug combination for less than 18 months; unfavourable responses were noted when ethambutol was omitted $(n=7) \cdot{ }^{13}$ Second-line drugs, including ethionamide and cycloserine, yielded poor results secondary to drug toxicity and poor compliance. ${ }^{13}$ In a second review, 19 cases of pulmonary infection due to $M$. malmoense were reported from Scotland..$^{14}$ All but one of the patients presented with cavitating pulmonary infection, and most of the patients had a predisposing lung condition; 17 were cigarette smokers. ${ }^{14}$ All patients received a standard drug regimen of rifampicin, isoniazid, and ethambutol with or without pyrazinamide. A range of outcomes were observed, but the responses were more satisfactory when ethambutol was included and when the regimen was continued for an extended period (mean 15 months). ${ }^{14}$

The first randomized trial of treatments for pulmonary disease caused by $M$. malmoense was completed by the British Thoracic Society, which compared regimens of rifampicin and ethambutol with rifampicin, ethambutol, and isoniazid. ${ }^{15} \mathrm{~A}$

\section{Table 1. Clinical and Microbiologic Criteria for Diagnosis of Nontuberculous Mycobacterial Pulmonary Disease ${ }^{1}$}

Type of Criteria

Clinical (both 1 AND 2 required)
Description

1. Pulmonary symptoms on chest auscultation (rhonchi, crackles, wheezes, or squeaks) combined with nodular or cavitary opacities on chest radiography OR

High-resolution computed tomography showing multifocal bronchiectasis with multiple small nodules

2. Appropriate exclusion of other diagnoses (e.g., tuberculosis, pulmonary malignancy)

Microbiologic (one OR more)
3. Positive culture from at least 2 sputum samples

4. Positive culture from at least 1 bronchial wash or lavage

5. Transbronchial or other lung biopsy with mycobacterial histopathologic findings (granulomatous inflammation or acid-fast bacilli) and positive culture for nontuberculous mycobacteria 
total of 106 patients with pulmonary infection due to $M$. malmoense were treated for 2 years. Rifampicin and ethambutol yielded cure rates similar to those achieved with rifampicin, ethambutol, and isoniazid. ${ }^{15}$ At 5-year follow-up, a total of $63(59 \%)$ patients were still alive, of whom 44 (20 receiving rifampicin and ethambutol and 24 receiving rifampicin, ethambutol, and isoniazid) were known to be cured. ${ }^{16}$ The authors concluded that the addition of isoniazid did not confer any therapeutic advantage. In addition, the rifampicin, ethambutol, and isoniazid regimen was associated with a higher death rate overall for all species studied, although this difference was nonsignificant. ${ }^{15,16}$

Finally, several reports have documented some success with the addition of fluoroquinolones and macrolides to the rifampicin and ethambutol regimen.-9,17 For example, the authors of a case report from British Columbia documented success when they added clarithromycin to the rifampicin and ethambutol regimen; the clarithromycin had a low minimum inhibitory concentration for $M$. malmoense and was generally well tolerated. ${ }^{6}$ In a more recent review of Dutch patients with M. malmoense pulmonary isolates who were seen between 2002 and 2006, 30 patients started treatment, macrolides were added for 22 patients (clarithromycin for 18 patients and azithromycin for 4), and fluoroquinolones were added for 6 patients (ciprofloxacin for 4 patients and moxifloxacin for 2). Notably, the overall response rate was $70 \%$, but the response was lower among those receiving macrolide-containing regimens (43\% versus 63\%), although this difference was not statistically significant. ${ }^{18}$

In summary, pulmonary disease due to $M$. malmoense and other NTM species is still most common in Europe but is increasingly becoming a problem in North America. Familiarity with the unique challenges in the diagnosis and treatment of these infections should improve the outcomes of patients in the short term. Unfortunately, the outcome was poor for the patient described here because of several contributing factors: extensive lung involvement, delay in diagnosis and initiation of treatment, significant malnutrition, Crohn disease, and superimposed pneumonia. Although unsuccessful in this case, a multiple-drug regimen incorporating ethambutol and a rifamycin administered for an extended period continues to provide the best chance of cure for $M$. malmoense disease. The case reported here highlights the difficulties in managing this disease, which can lead to unfortunate complications, including death. Further studies are needed to evaluate new drugs and new drug combinations targeting $M$. malmoense.

\section{References}

1. Griffith DE, Aksamit T, Brown-Elliott BA, Catanzaro A, Daley C, Gordin F, et al.; ATS Mycobacterial Diseases Subcommittee; American Thoracic Society; Infectious Disease Society of America. An official ATS/IDSA statement: diagnosis, treatment, and prevention of nontuberculous mycobacterial diseases. Am J Respir Crit Care Med 2007;175(4):367-416. Erratum in: Am J Respir Crit Care Med 2007;175(7):744-745 (dosage error in article text).

2. Schröder KH, Juhlin I. Mycobacterium malmoense sp. nov. J Syst Bacteriol 1977;27(3):241-246.
3. Hoefsloot W, Boeree MJ, van Ingen J, Bendien S, Magis C, de Lange W, et al. The rising incidence and clinical relevance of Mycobacterium malmoense: a review of the literature. Int J Tuberc Lung Dis 2008;12(9): 987-993.

4. Buchholz UT, McNeil MM, Keyes LE, Good RC. Mycobacterium malmoense infections in the United States, January 1993 through June 1995. Clin Infect Dis 1998;27(3):551-558.

5. Warren NG, Body BA, Silcox VA, Matthews JH. Pulmonary disease due to Mycobacterium malmoense. J Clin Microbiol 1984;20(2):245-247.

6. Al-Moamary MS, Black W, Elwood K. Pulmonary disease due to Mycobacterium malmoense in British Columbia. Can Respir J 1998; 5(2):135-138

7. Marras TK, Chedore P, Ying AM, Jamieson F. Isolation prevalence of pulmonary non-tuberculous mycobacteria in Ontario, 1997-2003. Thorax 2007;62(8):661-666.

8. McCrossin C, Mailman T. First Canadian reports of cervical adenitis due to Mycobacterium malmoense and a 10-year review of nontuberculous mycobacterial adenitis. Can J Infect Dis Med Microbiol 2006;17(2): 123-127.

9. Zaugg M, Salfinger M, Opravil M, Lüthy R. Extrapulmonary and disseminated infections due to Mycobacterium malmoense: case report and review. Clin Infect Dis 1993;16(4):540-549.

10. Hoffner SE, Hjelm U, Källenius G. Susceptibility of Mycobacterium malmoense to antibacterial drugs and drug combinations. Antimicrob Agents Chemother 1993;37(6):1285-1288.

11. Subcommittee of the Joint Tuberculosis Committee of the British Thoracic Society. Management of opportunist mycobacterial infections: Joint Tuberculosis Committee guidelines 1999. Thorax 2000;55(3):210-218.

12. Field SK, Cowie RL. Lung disease due to more common nontuberculous mycobacteria. Chest 2006;129(6):1653-1672.

13. Banks J, Jenkins PA, Smith AP. Pulmonary infection with Mycobacterium malmoense- a review of treatment and response. Tubercle 1985;66(3):197-203.

14. France AJ, McLeod DT, Calder MA, Seaton A. Mycobacterium malmoense infections in Scotland: an increasing problem. Thorax 1987;42(8):593-595.

15. Research Committee of the British Thoracic Society. First randomized trial of treatments for pulmonary disease caused by $M$ avium intracellulare, $M$ malmoense, and $M$ xenopi in HIV negative patients: rifampicin, ethambutol and isoniazid versus rifampicin and ethambutol. Thorax 2001;56(3):167-172.

16. Research Committee of the British Thoracic Society. Pulmonary disease caused by $M$. malmoense in HIV negative patients: 5-yr follow-up of patients receiving standardized treatment. Eur Respir J 2003;21(3):478-482.

17. Enzensberger R, Hunfeld KP, Krause M, Rüsch-Gerdes S, Brade V, Böddinghaus B. Mycobacterium malmoense infections in immunocompetent patients. Eur J Clin Microbiol Infect Dis 1999;18(8):579-581.

18. Hoefsloot W, van Ingen J, de Lange WC, Dekhuijzen PN, Boeree MJ, van Soolingen D. Clinical relevance of Mycobacterium malmoense isolation in the Netherlands. Eur Respir J 2009;34(4);925-931.

Cory D Cowan, BSP, PharmD, was, at the time of writing, a PharmD candidate with the University of Toronto. He is now the Product Manager, Business Development, for ESI Canada in Mississauga, Ontario.

John J Hawboldt, BSP, ACPR, PharmD, is Associate Professor, School of Pharmacy, Memorial University of Newfoundland Health Sciences Center, St John's, Newfoundland and Labrador.

Mazen Bader, MD, MPh, is Assistant Professor of Medicine in the Department of Medicine, Division of Infectious Diseases, McMaster University-Henderson Site, Henderson General Hospital, Hamilton, Ontario.

\section{Address correspondence to:}

Dr Cory D Cowan

Product Manager, Business Development

ESI Canada

5770 Hurontario Street

10th Floor

Mississauga ON L5R 365

e-mail: ccowan@express-scripts.com 